Review Article

\title{
Dual-Tracer Positron-Emission Tomography Using Prostate-Specific Membrane Antigen and Fluorodeoxyglucose for Staging of Prostate Cancer: A Systematic Review
}

\author{
Stephen McGeorge $\mathbb{D}^{1,2,3}$ Michael Kwok ${ }^{1},{ }^{2,3}$ Andrew Jiang, ${ }^{3}$ Louise Emmett ${ }^{\mathbb{D}},{ }^{4,5}$ \\ David A. Pattison (D),,$^{3,6}$ Paul A. Thomas $(\mathbb{D})^{3,6}$ John W. Yaxley (D), ${ }^{1,3,7}$ \\ and Matthew J. Roberts $\mathbb{D}^{1,2,3,8}$ \\ ${ }^{1}$ Department of Urology, Royal Brisbane and Women's Hospital, Butterfield St, Herston 4029, Queensland, Australia \\ ${ }^{2}$ Department of Urology, Redcliffe Hospital, Anzac Avenue, Redcliffe 4020, Queensland, Australia \\ ${ }^{3}$ Faculty of Medicine, The University of Queensland, Herston 4006, Queensland, Australia \\ ${ }^{4}$ Department of Theranostics and Nuclear Medicine, St. Vincent's Hospital Sydney, 390 Victoria St, Darlinghurst 2010, \\ New South Wales, Australia \\ ${ }^{5}$ Garvan Institute of Medical Research, 384 Victoria St, Darlinghurst 2010, New South Wales, Australia \\ ${ }^{6}$ Department of Nuclear Medicine \& Specialised PET Services, Royal Brisbane and Women's Hospital, Butterfield St, \\ Herston 4029, Queensland, Australia \\ ${ }^{7}$ Wesley Urology Clinic, Wesley Hospital, 451 Coronation Dr, Auchenflower 4066, Queensland, Australia \\ ${ }^{8}$ University of Queensland Centre for Clinical Research, Faculty of Medicine, Building 71/918 RBWH, Butterfield St, \\ Herston 4029, Queensland, Australia
}

Correspondence should be addressed to Matthew J. Roberts; m.roberts2@uq.edu.au

Received 9 June 2021; Revised 29 July 2021; Accepted 6 August 2021; Published 18 August 2021

Academic Editor: Mohammad H. Ather

Copyright ( $\odot 2021$ Stephen McGeorge et al. This is an open access article distributed under the Creative Commons Attribution License, which permits unrestricted use, distribution, and reproduction in any medium, provided the original work is properly cited.

PSMA PET is more accurate than conventional imaging (CT/bone scan) for staging of intermediate- or high-risk prostate cancer (PCa), but 5-10\% of primary tumours have low PSMA ligand uptake. FDG PET has been used to further define disease extent in endstage castrate-resistant PCa and may be beneficial earlier in the disease course for more accurate staging. The objective of this study was to review the available evidence for patients undergoing both FDG and PSMA PET for PCa staging at initial diagnosis and in recurrent disease. A systematic literature review was performed for studies with direct, intraindividual comparison of PSMA and FDG PET for staging of PCa. Assessment for radioligand therapy eligibility was not considered. Risk of bias was assessed. 543 citations were screened and assessed. 13 case reports, three retrospective studies, and one prospective study were included. FDG after PSMA PET improved the detection of metastases from $65 \%$ to $73 \%$ in high-risk early castration-resistant PCa with negative conventional imaging (M0). Positive FDG PET was found in 17\% of men with negative PSMA PET for postprostatectomy biochemical recurrence. Gleason score $\geq 8$ and higher PSA levels predicted FDG-avid metastases in BCR and primary staging. Variant histology (ductal and neuroendocrine) was common in case reports, resulting in PSMA-negative FDG-positive imaging for 3 patients. Dual-tracer PET for PCa may assist in characterising high-risk disease during primary staging and restaging. Further studies are required to determine the additive benefit of FDG PET and if the FDG-positive phenotype may indicate a poorer prognosis.

\section{Introduction}

Prostate cancer $(\mathrm{PCa})$ is the most commonly diagnosed internal malignancy and high-ranking cause of mortality in men worldwide $[1,2]$. An increased incidence of de novo metastatic PCa has been observed since 2004, most significantly in men aged 55 to 69 years, potentially contributing to higher mortality $[3,4]$. Modern approaches to metastatic PCa treatment incorporate aggressive systemic therapy, such as taxane-based chemotherapy, androgen receptor 
inhibitors, and radioligand therapy (RLT) [5, 6]. Additionally, earlier use of systemic therapy prior to treatment of high-risk, locally advanced disease has been reported and is the subject of ongoing clinical trials [7-10]. However, accurate patient selection for systemic therapy remains one of the key challenges in urologic oncology.

Despite limitations, conventional imaging with computed tomography (CT) and technetium-based bone scan are widely used for staging [11]. However, cumulative evidence indicates that prostate-specific membrane antigen (PSMA) positron-emission tomography (PET) should be a centrepiece of staging for intermediate- to high-risk patients [11-16]. A prospective, randomised trial by Hofman and colleagues determined that PSMA PET/CT was 27\% (95\% CI: 23-31) more accurate than conventional imaging [12]. However, $5-10 \%$ of primary PCa tumours have low PSMA activity which evade detection by PSMA PET, mostly in high-grade and variant tumour types [14-18].

18-F-Fluorodeoxyglucose (FDG) PET use in PCa has previously been of limited benefit in primary staging and biochemical recurrence (BCR) $[19,20]$. In a study of 41 men with Gleason score $\geq 8 \mathrm{PCa}$ who had FDG PET/CT for primary staging, 11 men had nodal metastasis from histopathology, and only 3 of these men (27\%) had corresponding lymph node FDG uptake [21]. Increasing use of FDG PET to aid patient selection for RLT and other therapies in $\mathrm{mCRPCa}$ has provided insight into tumour heterogeneity and benefits of dual-tracer PET imaging [22, 23]. Discordant findings have been attributed to the increased anaerobic glycolysis detected by FDG PET in patients with more aggressive histological types [24]. Whilst the use of PSMA and FDG PET imaging in PCa has been examined individually, the potential diagnostic impact for individual patients to undergo dual-tracer PET imaging remains unclear. Additional use of FDG PET may improve disease characterisation in patients with high-risk localised and metastatic $\mathrm{PCa}$, with inconclusive conventional imaging and negative PSMA PET.

We sought to summarise the available evidence for the use of dual-tracer PET, being 18-F-fluorodeoxyglucose (FDG) PET in addition to PSMA PET, for PCa staging at initial diagnosis and in recurrent disease.

\section{Methods}

2.1. Search Strategy. A systematic literature search was performed in April 2021 (Table S1) in accordance with the preferred reporting items for systematic reviews and metaanalysis (PRISMA) and the Cochrane Handbook guidelines $[25,26]$. The review protocol was registered (CRD42020201307) and published in the International Prospective Register of Systematic Reviews (PROSPERO). Citation searches were conducted from included reports to ensure all relevant studies were captured. All included studies received ethical approval, and the source data were publicly available, so ethical approval was not sought [27].

2.2. Study Selection. All original research reports that directly compared PSMA and FDG PET in the setting of histologically diagnosed or clinically suspected PCa were considered. Editorials, review articles, and animal studies were excluded, as were studies comparing PSMA and FDG PET for the purpose of RLT, as this topic was felt to be beyond the scope of the current review. Furthermore, records were excluded if they assessed other primary malignancies or active systemic disease accounting for tracer uptake, PCa was not specifically diagnosed, or they compared alternative tracers that were not FDG or PSMA.

2.3. Quality Assessment. Methodological quality was appraised using checklists recommended by the Cochrane Guidelines [28, 29]. Each article was assessed for bias by two independent authors, with a third reviewer used to resolve disagreements (Tables S2 and S3).

2.4. Data Management. The data from case reports included, where available, patient age, PSA at the time of imaging, indication for dual-tracer use, sites of metastatic lesions, ligand uptake patterns for FDG and PSMA, previous PCa treatments, histological PCa type, and Gleason score. Cases were considered to be positive if ligand uptake (according to criteria used in each case) was reported in either the prostate or at sites of metastatic disease. Discordant ligand uptake was reported when both FDG and PSMA PET were positive, but there were different sites of the disease. If multiple PSA values were reported, the PSA at the time of PET imaging was used for this comparison. For articles concerning a population, these data were collected as a range, where applicable, as well as the inclusion and exclusion criteria.

Included studies displayed significant heterogeneity and small overall population, so a quantitative assessment was not possible (Table S4).

\section{Results}

The database search yielded 543 citations. Following screening and assessment (Figure 1), one single-arm prospective trial, three retrospective observational studies, and 13 case reports were included in the final analysis (Tables 1 and 2).

3.1. Castration-Resistant Disease. A prospective single-arm trial investigated the prevalence of PSMA-negative FDGpositive lesions in 37 men with negative conventional imaging (M0) in the setting of high-risk early castration resistance (defined as PSA progression with PSA $\leq 2 \mathrm{ng} / \mathrm{ml}$ and PSA doubling time $\leq 10$ months) who were restaged using PSMA and then FDG PET [33]. 65\% of men had metastatic disease on initial PSMA PET, while an additional 5\% had localised PSMA avidity within the prostate, which was concordant with FDG PET. Among 30\% of men with no PSMA avidity, $27 \%$ ( $8 \%$ overall) showed metastatic disease according to FDG PET, increasing the detection of metastases from $65 \%$ to $73 \%$.

High-grade disease, defined as Gleason grade group of four or higher, correlated with nodal or distant metastases on FDG PET $(\mathrm{OR}=13.09, p=0.02)$. A PSA doubling time less 


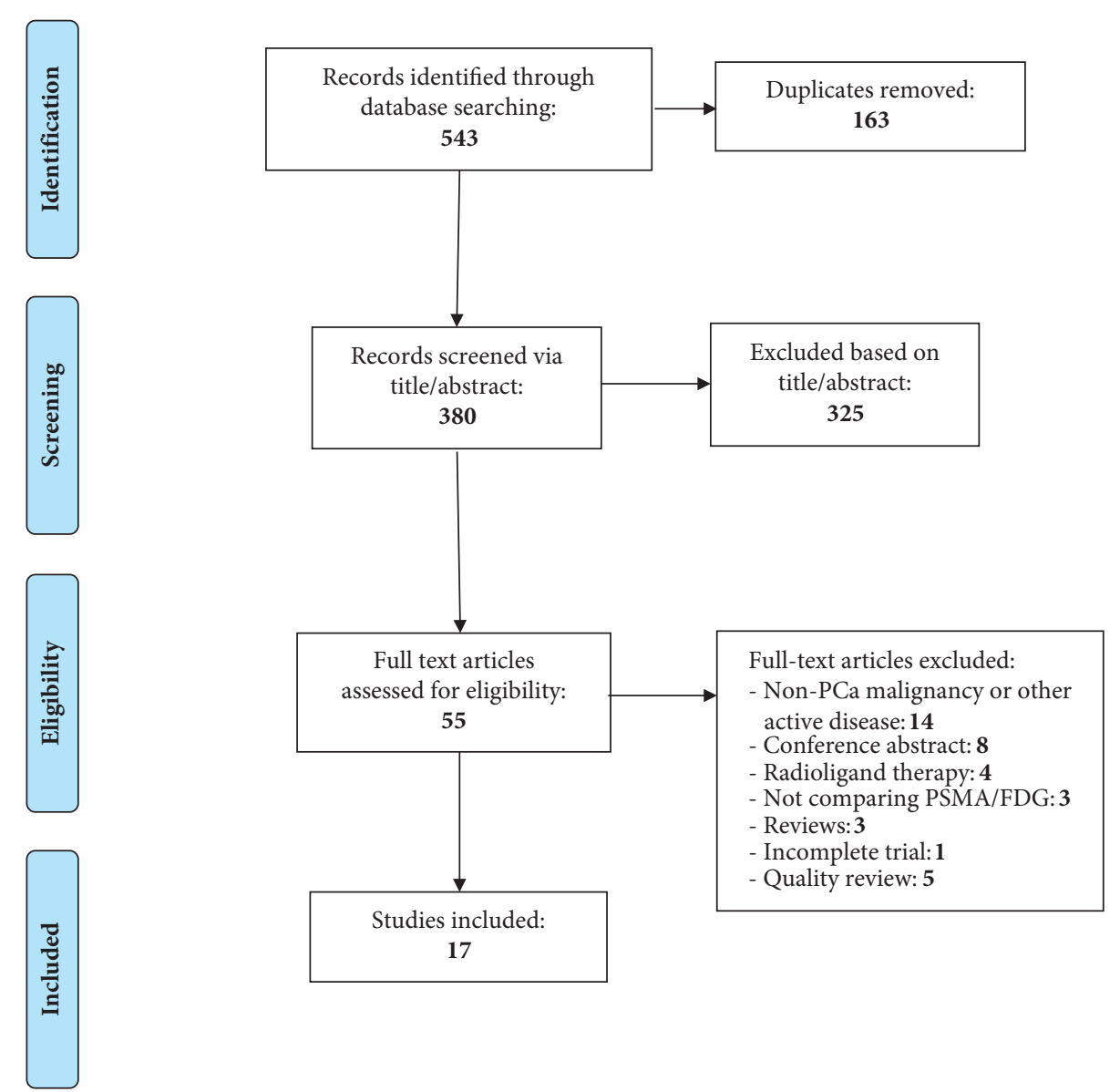

Figure 1: PRISMA 2009 flow diagram.

than six months was associated with the presence of PSMApositive metastatic disease ( $O R=8.00, p=0.009)$, but not for FDG-positive metastases $(p=0.29)$. Discordant lesions (PSMA-negative FDG-positive) were more common for axial bone lesions compared to concordant PSMA-FDG-positive patients $(p=0.02)$. Metastatic disease burden was according to CHAARTED criteria and was unchanged in the use of FDG PET [47]. However, use of FDG PET was estimated to enable an additional six men (19 in total) precise lesion targeting for oligometastatic-directed therapy according to SABR-COMET trial criteria [48].

Within the same manuscript, an additional analysis of a retrospective cohort of 41 men was reported. The cohort included 14 men with BCR, 18 men with hormone-sensitive PCa (HSPCa), and 9 men with mCRPCa. Metastatic disease according to PSMA PET was detected in $29 \%, 39 \%$, and $100 \%$ of men (with FDG concordant disease for $100 \%, 86 \%$, and $67 \%$ ) with BCR, HSPCa, and MCRPCa, respectively. PSMA-negative FDG-positive disease was rare in HSPCa (6\%) and more commonly observed for CRPCa (33\%).

3.2. Biochemically Recurrent Hormone-Sensitive Disease. Predictive factors for FDG-positive PET in men with BCR (PSA $>0.2 \mathrm{ng} / \mathrm{ml}$ ) after radical prostatectomy and a negative PSMA PET were determined in a retrospective case-control study $(n=72)$ [32]. The median PSA was $3.4 \mathrm{ng} / \mathrm{ml}$, and $53 \%$ had prior adjuvant therapy. FDG PET was positive in $17 \%$ of men, where serum PSA (16.7 versus $0.8 \mathrm{ng} / \mathrm{ml}$ ) and Gleason scores $(34.4 \%$ with Gleason score $\geq 8$ vs. $2.5 \%$ with Gleason score $<8 ; p<0.001)$ were higher than the FDG-negative group. There were no differences in age, PSA doubling time, interval between PSMA and FDG PET, or adjuvant therapies. A PSA cutoff of $2.3 \mathrm{ng} / \mathrm{ml}$ was $91.7 \%$ accurate for FDG PET positivity (area under the curve of $0.872, p<0.001$ ). The group was divided into three groups for the prediction of positive FDG in the setting of negative PSMA, being low potential (PSA $<2.3 \mathrm{ng} / \mathrm{ml}$ and Gleason score $<8$ ), moderate potential (PSA $\geq 2.3 \mathrm{ng} / \mathrm{ml}$ or Gleason score $\geq 8$, but not both), and high potential (PSA $\geq 2.3 \mathrm{ng} / \mathrm{ml}$ and Gleason score $\geq 8)$. These groups showed $0 \%(36 / 36), 11.5 \%(3 / 26)$, and 90\% (9/10) positive FDG PET results, respectively.

A retrospective evaluation of 138 men who underwent ${ }^{68}$ Ga-PSMA-11 and FDG PET for differentiating lymph node metastases from peripheral ganglia was examined for oncological outcomes [31]. Imaging was for primary staging in $47 \%(n=65)$ and BCR in $53 \%(n=73) .47 \%$ of men had Gleason $\geq 8$ disease, and mean PSA was $56.4 \mathrm{ng} / \mathrm{mL}$ (IQR: 18.5-99.7) and $1.1 \mathrm{ng} / \mathrm{mL}$ (IQR: $0.4-4.1$ ) in the primary staging and BCR cohorts, respectively. $42 \%$ of patients demonstrated PSMA-avid lymph node metastases, totalling 83 metastases, with FDG concordance in 63\% $(n=52)$. 


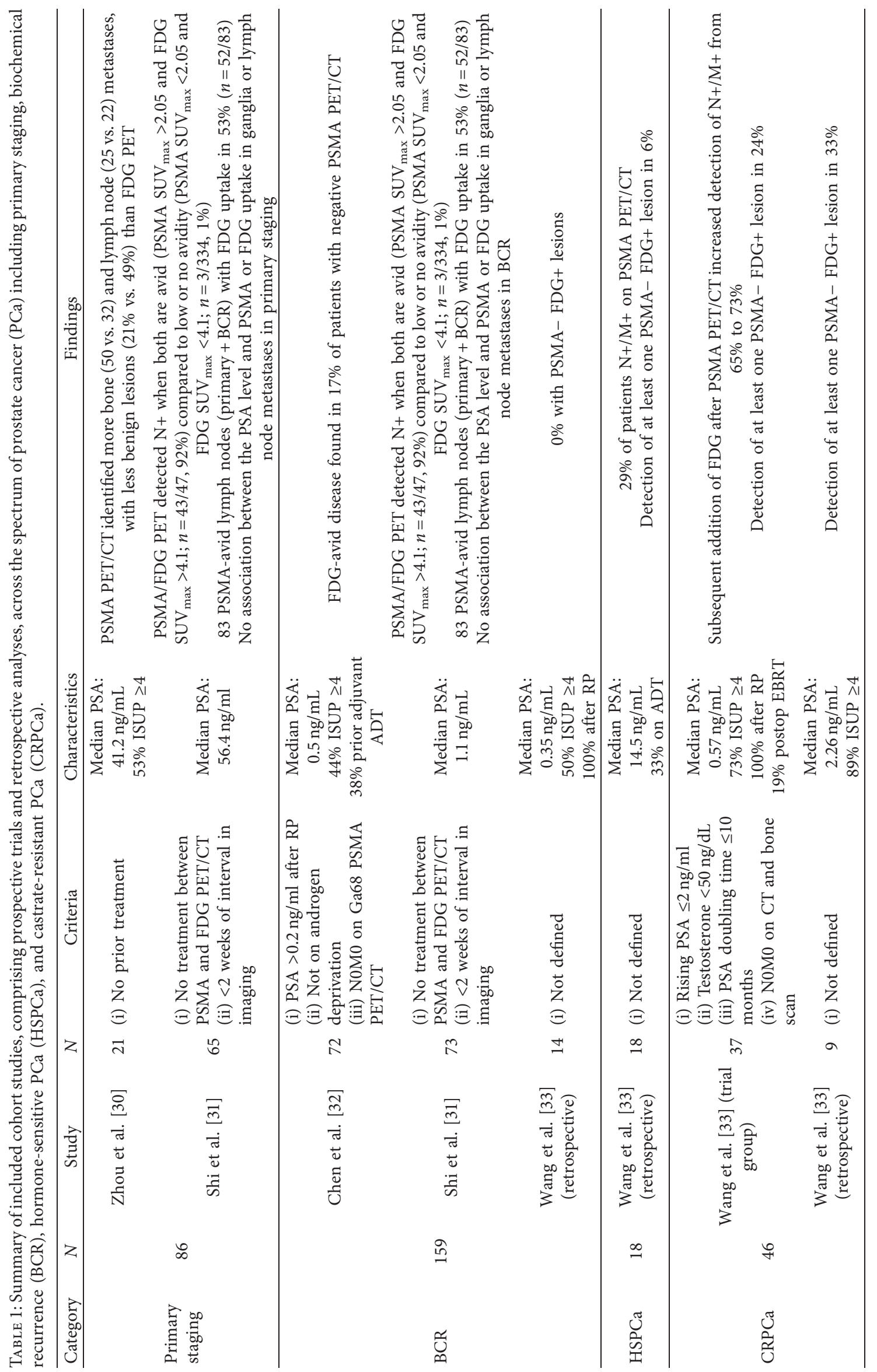




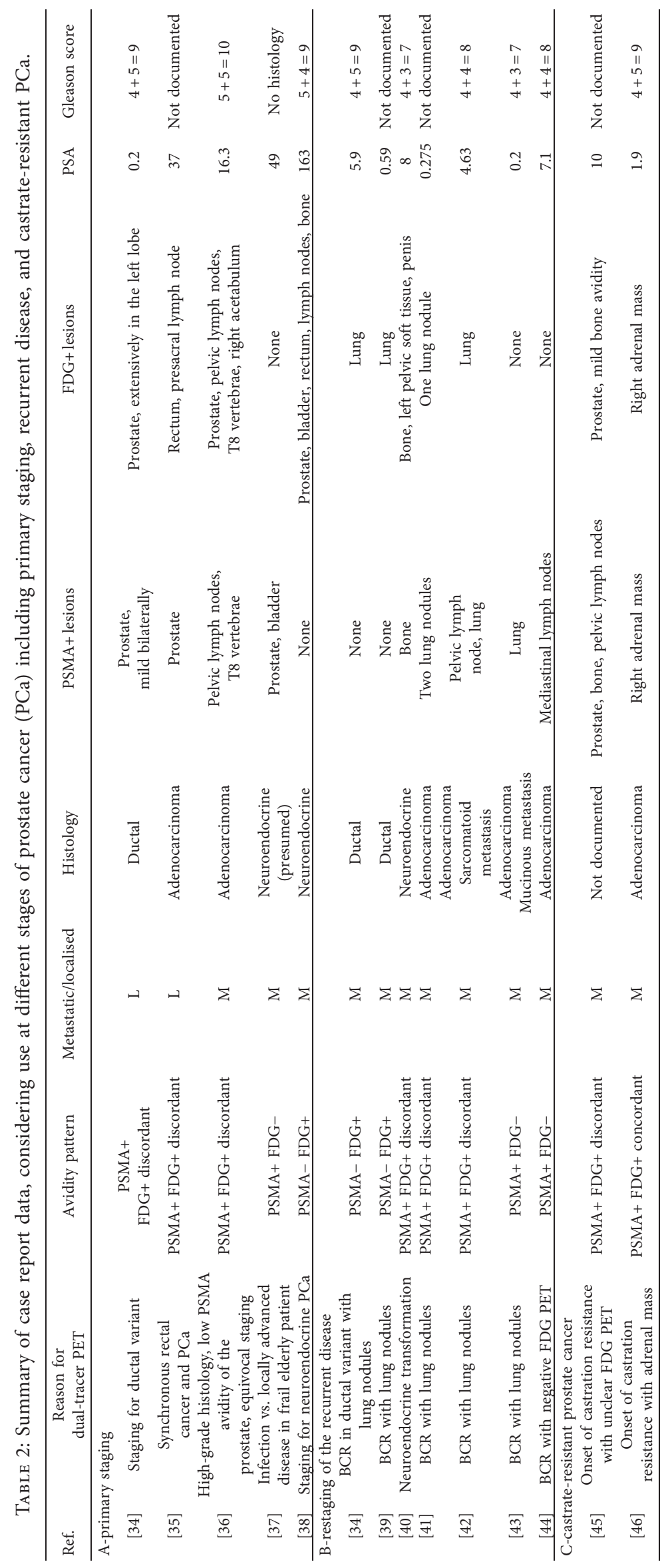


Significantly less ganglia showed FDG uptake $(p<0.001)$, so the PSMA/FDG PET combination was shown to detect lymph node metastases when both were avid (PSMA $\mathrm{SUV}_{\text {max }}>2.05$ and FDG SUV max $\left.>4.1 ; 43 / 47,92 \%\right)$ compared to low or no avidity (PSMA SUV max $_{2}<2.05$ and FDG $\left.\mathrm{SUV}_{\max }<4.1 ; 3 / 334,1 \%\right)$. No correlation was found for PSMA or FDG uptake (according to $\mathrm{SUV}_{\max }$ ) with Gleason scores or PSA level (binary grouping, not defined, $p>0.05$ ) in both primary staging and BCR cohorts.

3.3. Primary Staging. Staging with dual-tracer PSMA/FDG $\mathrm{PET} / \mathrm{CT}$ prior to treatment was examined in one retrospective study of 21 men [30]. 68.7\% $(n=11 / 16)$ of men had Gleason scores $\geq 8$, and median PSA at diagnosis was $41.2 \mathrm{ng} / \mathrm{mL}$ (range: 5-200). All patients had ${ }^{18} \mathrm{~F}$-PSMA1007 uptake within the prostate; however, $66.7 \%$ had local FDG uptake. PSMA PET/CT identified more bone (50 vs. 32) and lymph node (25 vs. 22) metastases, with less benign lesions (21\% vs. $49 \%$ ) than FDG PET. Most patients with metastases on FDG PET had Gleason scores

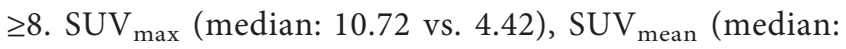
6.67 vs. 2.59 ), and tumour-to-background ratio (13.3 vs. 7.91) of identified metastases were all higher for ${ }^{18} \mathrm{~F}$ PSMA-1007 than FDG PET $(p<0.001)$.

3.4. Case Reports. Case report data are summarised in Table 2 (Ref. A-C) according to indication for dual-tracer use with subgrouping according to the ligand uptake pattern. The most common indication was for men with BCR requiring characterisation of lung nodules seen on conventional imaging (5/14 patients) [34, 39, 41-43]. Variant histology was also common, with three cases of ductal variant and three cases of neuroendocrine differentiation, although one of these was presumed on the basis of a raised serum chromogranin A level in combination with DOTATOC PET avidity [34, 37-40].

PSMA and FDG ligand uptake patterns were discordant in the majority of cases, although this was not consistently reported for each individual lesion in every patient (Table 2). Three cases reported a negative PSMA PET with positive FDG PET, all of which had either ductal or neuroendocrine histology. Serum PSA levels were highly variable for all patterns of ligand uptake according to the tracer, being $0.2-37 \mathrm{ng} / \mathrm{ml}$ for discordant PSMA-positive FDG-positive, $0.2-49 \mathrm{ng} / \mathrm{ml}$ for PSMA-positive FDG-negative, and $0.59-163 \mathrm{ng} / \mathrm{ml}$ in PSMA-negative FDG-positive.

\section{Discussion}

The role of dual-tracer PET for localised or recurrent PCa is unclear, and available data are limited. Despite significant heterogeneity in patient populations, indications, and performance which limit meta-analysis, discordant imaging and altered staging classification were observed. Imaging discordance was more likely to be observed in more advanced disease, presumably due to greater tumour clone heterogeneity.
An important application of dual-tracer PET/CT may be in the identification of additional sites of disease amenable to oligometastatic directed therapy. In the early castrate-resistant population discussed by Wang et al., 24\% of patients had at least one PSMA- FDG+ lesion which would have been otherwise missed [33]. The SABR-COMET trial found an overall survival benefit with stereotactic ablative radiotherapy, and results from the ORIOLE trial showed that the total consolidation of all PSMA-avid diseases gave significant progression-free survival and distant metastasis-free survival advantages [48, 49]. Therefore, dual PSMA/FDG PET/CT may improve oncological outcomes by identifying additional sites of the disease amenable to oligometastatic directed therapy.

Furthermore, the PSMA-negative FDG-positive phenotype may represent a population at a greater risk of rapid disease progression. These poor oncological outcomes have been reported in the context of RLT, with Suman and colleagues reporting that, among 40 patients who underwent ${ }^{177}$ Lu-PSMA-617 RLT, high FDG uptake $\left(\mathrm{SUV}_{\max }>15\right)$ was reported to correlate with worse 12-month progression-free survival (PFS; $p=0.05$ ) and poor response to RLT (disease progression in 12/15 patients) [50]. Furthermore, a study of 16 men with poor PSMA avidity or significantly discordant FDG avidity ineligible for ${ }^{177}$ Lu-PSMA-617 RLT reported a median overall survival of 2.3 months when FDG avidity was high [51]. While PSMA-negative imaging may suggest aggressive disease, a lower ratio of PSMA SUV $\max$ to FDG $\mathrm{SUV}_{\text {max }}$ may better predict PSA reduction $\geq 30 \%$ following RLT $(p<0.02)$ [23]. However, FDG findings alone were not predictive of response, and no imaging factors correlated with PSA response $\geq 50 \%$. Conversely, $59 \%$ of men with low FDG uptake $\left(\mathrm{SUV}_{\max }<15\right)$ reported improved or stable disease with RLT in one study, while absent PSMA and FDG avidity following RLT was indicative of favourable treatment response [50].

We observed low PSMA avidity in FDG-positive disease to be a common finding among papers included in this review. Low PSMA avidity in advanced PCa is hypothesised to be due to dedifferentiation or neuroendocrine transformation. Biomarker-focused research supports this hypothesis, with a retrospective analysis in 66 patients referred for RLT, of which 41 patients (62\%) had at least one PSMAnegative FDG-positive lesion and demonstrated higher levels of neuron-specific enolase, a neuroendocrine marker [52]. Furthermore, neuroendocrine PCa has a more aggressive course and thereby an expectation of increased anaerobic glycolysis with consequent FDG avidity [24]. This is in keeping with existing evidence on divergent clonal evolution of CRPCa and the "genomic overlap" between conventional adenocarcinoma and neuroendocrine PCa in the development of castration resistance [53]. There is also support in the correlation between levels of glucose uptakeassociated genes with neuroendocrine gene signatures and low PSMA expression [54]. Given the poor prognosis in these patients, further research on dual-tracer PET may help streamline earlier diagnosis of this $\mathrm{PCa}$ phenotype and thereby prompt earlier escalation to more aggressive treatment to improve oncological outcomes. 
PSMA PET has been shown to be superior to conventional imaging for the initial staging of intermediate- and high-risk PCa [12]. However, approximately 5-10\% of primary PCa has insufficient PSMA avidity for PET detection due to contributions of both low- (usually of lower volume) and high-grade disease [14]. In the latter population, the available data suggest that FDG may detect primary and metastatic disease that would otherwise not be observed on PSMA PET or conventional imaging. Thus, the potential for FDG PET use in the primary staging of highgrade $\mathrm{PCa}$, especially with neuroendocrine or ductal variation, may be an early independent identifier of aggressive disease and poorer oncological outcomes with conventional therapies.

The limitations of this review include limited available studies and details of data included in these studies, including their mostly retrospective nature and limited coverage of each disease state. Furthermore, we were unable to adjust for selection and publication bias in the included studies. Conversely, to our knowledge, this review represents the first summary of the available literature of the intraindividual assessment of PSMA and FDG PET.

In conclusion, the diagnostic utility of dual-tracer FDG/PSMA PET/CT for PCa may assist in characterising high-risk disease during primary staging and restaging. Addition of FDG PET can identify additional sites of the disease amenable to oligometastatic directed therapy. $\mathrm{Pa}$ tients with FDG-positive disease in advanced PCa states have a poor prognosis, especially with concurrently negative PSMA PET. When applied to high-risk (high-grade or variant histology), hormone-sensitive disease in the localised or recurrent stage, detection of the FDG-positive phenotype may signal a poorer prognosis to prompt more aggressive intervention earlier in the disease course. Further studies are required to validate the prognostic insights outlined here and selective incorporation into therapeutic clinical trials.

\section{Data Availability}

The search strategies for each database used for the systematic review are included in the supplementary data. The PRISMA flowchart shows inclusion/exclusion of citations. Citations excluded in screening are held in a Covidence database by the authors and can be made available if required. Articles excluded by quality review are listed in the supplementary materials [55-59].

\section{Disclosure}

An earlier version of this systematic review was presented as a poster at the Royal Australasian College of Surgeons Annual Scientific Congress, Melbourne, Victoria, Australia, 10-14 May 2021, and abstract published in ANZ J Surg. 2021 May; 91 Suppl 1: 259-289.

\section{Conflicts of Interest}

The authors declare that there are no conflicts of interest.

\section{Acknowledgments}

MJR was supported by a Clinician Research Fellowship from the Metro North Office of Research, Queensland Health, and a Doctor in Training Research Scholarship from Avant Mutual Group Pty Ltd.

\section{Supplementary Materials}

Table S1: search strategy by the database. Table S2: risk of bias assessment using QUADAS-2. Table S3: risk of bias assessment for case reports, Joanna Briggs Institute Checklist for Case Reports. Table S4: summary of articles. (Supplementary Materials)

\section{References}

[1] C. H. Pernar, E. M. Ebot, K. M. Wilson, and L. A. Mucci, "The epidemiology of prostate cancer," Cold Spring Harbor Perspectives in Medicine, vol. 8, no. 12, 2018.

[2] Global Burden of Disease Cancer Collaboration, C. Fitzmaurice, C. Allen et al., "Global, regional, and national cancer incidence, mortality, years of life lost, years lived with disability, and disability-adjusted life-years for 32 cancer groups, 1990 to 2015: a systematic analysis for the global burden of disease study," JAMA Oncology, vol. 3, no. 4, pp. 524-548, 2017.

[3] S. Smith and P. Wolanski, "Metastatic prostate cancer incidence in Australia after amendment to prostate-specific antigen screening guidelines," ANZ Journal of Surgery, vol. 88, no. 7-8, pp. E589-E593, 2018.

[4] A. B. Weiner, R. S. Matulewicz, S. E. Eggener, and E. M. Schaeffer, "Increasing incidence of metastatic prostate cancer in the United States (2004-2013)," Prostate Cancer and Prostatic Diseases, vol. 19, no. 4, pp. 395-397, 2016.

[5] M. Perera, M. J. Roberts, L. Klotz et al., "Intermittent versus continuous androgen deprivation therapy for advanced prostate cancer," Nature Reviews Urology, vol. 17, no. 8, pp. $469-481,2020$.

[6] P. Cornford, R. C. N. Van den Bergh, E. Briers et al., "EAUEANM-ESTRO-ESUR-SIOG guidelines on prostate cancer. Part II-2020 update: treatment of relapsing and metastatic prostate cancer," European Urology, vol. 79, no. 2, pp. 263-282, 2021.

[7] J. A. Eastham, G. Heller, S. Halabi et al., "Cancer and leukemia group B 90203 (alliance): radical prostatectomy with or without neoadjuvant chemohormonal therapy in localized, high-risk prostate cancer," Journal of Clinical Oncology, vol. 38, no. 26, pp. 3042-3050, 2020.

[8] B. Montgomery, M. S. Tretiakova, A. M. Joshua et al., "Neoadjuvant enzalutamide prior to prostatectomy," Clinical Cancer Research, vol. 23, no. 9, pp. 2169-2176, 2017.

[9] T. R. McClintock, N. Von Landenberg, A. P. Cole et al., "Neoadjuvant androgen deprivation therapy prior to radical prostatectomy: recent trends in utilization and association with postoperative surgical margin status," Annals of Surgical Oncology, vol. 26, no. 1, pp. 297-305, 2019.

[10] N. Dhiantravan, J. Violet, R. Eapen et al., "Clinical trial protocol for LuTectomy: a single-arm study of the dosimetry, safety, and potential benefit of (177)Lu-PSMA-617 prior to prostatectomy," European Urology Focus, vol. 7, no. 2, pp. 234-237, 2021. 
[11] M. S. Litwin and H.-J. Tan, "The diagnosis and treatment of prostate cancer," Journal of the American Medical Association, vol. 317 , no. 24, pp. 2532-2542, 2017.

[12] M. S. Hofman, N. Lawrentschuk, R. J. Francis et al., "Prostatespecific membrane antigen PET-CT in patients with high-risk prostate cancer before curative-intent surgery or radiotherapy (proPSMA): a prospective, randomised, multicentre study," The Lancet, vol. 395, no. 10231, pp. 1208-1216, 2020.

[13] P. Donato, M. J. Roberts, A. Morton et al., "Improved specificity with 68Ga PSMA PET/CT to detect clinically significant lesions "invisible" on multiparametric MRI of the prostate: a single institution comparative analysis with radical prostatectomy histology," European Journal of Nuclear Medicine and Molecular Imaging, vol. 46, no. 1, pp. 20-30, 2019.

[14] T. Maurer, M. Eiber, M. Schwaiger, and J. E. Gschwend, "Current use of PSMA-PET in prostate cancer management," Nature Reviews Urology, vol. 13, no. 4, pp. 226-235, 2016.

[15] M. J. Roberts, A. Morton, P. Donato et al., “68Ga-PSMA PET/ CT tumour intensity pre-operatively predicts adverse pathological outcomes and progression-free survival in localised prostate cancer," European Journal of Nuclear Medicine and Molecular Imaging, vol. 48, no. 2, pp. 477-482, 2021.

[16] T. Lengana, I. O. Lawal, T. G. Boshomane et al., "68Ga-PSMA $\mathrm{PET} / \mathrm{CT}$ replacing bone scan in the initial staging of skeletal metastasis in prostate cancer: a fait accompli?" Clinical Genitourinary Cancer, vol. 16, no. 5, pp. 392-401, 2018.

[17] A. Ghosh and W. D. W. Heston, "Tumor target prostate specific membrane antigen (PSMA) and its regulation in prostate cancer," Journal of Cellular Biochemistry, vol. 91, no. 3, pp. 528-539, 2004.

[18] P. S. Chakraborty, M. Tripathi, K. K. Agarwal, R. Kumar, M. K. Vijay, and C. Bal, "Metastatic poorly differentiated prostatic carcinoma with neuroendocrine differentiation," Clinical Nuclear Medicine, vol. 40, no. 2, pp. e163-e166, 2015.

[19] K. L. Wallitt, S. R. Khan, S. Dubash, H. H. Tam, S. Khan, and T. D. Barwick, "Clinical PET imaging in prostate cancer," RadioGraphics, vol. 37, no. 5, pp. 1512-1536, 2017.

[20] H. Jadvar, "Imaging evaluation of prostate cancer with $18 \mathrm{~F}-$ fluorodeoxyglucose PET/CT: utility and limitations," European Journal of Nuclear Medicine and Molecular Imaging, vol. 40, no. Suppl 1, pp. S5-S10, 2013.

[21] J.-M. Beauregard, A.-C. Blouin, V. Fradet et al., "FDG-PET/ CT for pre-operative staging and prognostic stratification of patients with high-grade prostate cancer at biopsy," Cancer Imaging, vol. 15, no. 1, p. 2, 2015.

[22] M. S. Hofman, J. Violet, R. J. Hicks et al., "[177 Lu]-PSMA-617 radionuclide treatment in patients with metastatic castrationresistant prostate cancer (LuPSMA trial): a single-centre, single-arm, phase 2 study," The Lancet Oncology, vol. 19, no. 6, pp. 825-833, 2018.

[23] L. Emmett, M. Crumbaker, B. Ho et al., "Results of a prospective phase 2 pilot trial of 177Lu-PSMA-617 therapy for metastatic castration-resistant prostate cancer including imaging predictors of treatment response and patterns of progression," Clinical Genitourinary Cancer, vol. 17, no. 1, pp. 15-22, 2019.

[24] M. P. Mannas, T. Lee, M. Pourghiasian, D. C. Wilson, and P. C. Black, "Incidentalomas of the prostate detected by $18-$ fluoro-2-deoxy-D-glucose positron emission tomography/ computed tomography," Canadian Urological Association Journal = Journal de l'Association des urologues du Canada, vol. 14, no. 5, pp. E180-E184, 2020.
[25] J. P. T. Higgins and Cochrane Collaboration, Cochrane Handbook for Systematic Reviews of Interventions, WileyBlackwell, Hoboken, NJ, USA, Second edition, 2020.

[26] D. Moher, A. Liberati, J. Tetzlaff, D. G. Altman, and P. Group, "Preferred reporting items for systematic reviews and metaanalyses: the PRISMA statement," PLoS Medicine, vol. 6, no. 7, Article ID e1000097, 2009.

[27] H. Suri, "Ethical considerations of conducting systematic reviews in educational research," in Systematic Reviews in Educational Research: Methodology, Perspectives and Application, O. Zawacki-Richter, M. Kerres, S. Bedenlier, M. Bond, and K. Buntins, Eds., Springer Fachmedien Wiesbaden, Wiesbaden, Germany, pp. 41-54, 2020.

[28] Joanna Briggs Institute, Checklist for Case Reports [The Joanna Briggs Institute Critical Appraisal Tools for Use in JBI Systematic Reviews], http://joannabriggs.org/assets/docs/criticalappraisal-tools/JBI_Critical_Appraisal-Checklist_for_Case_ Reports.pdf, Joanna Briggs Institute, Adelaide, Australia, 2016, http://joannabriggs.org/assets/docs/critical-appraisaltools/JBI_Critical_Appraisal-Checklist_for_Case_Reports. pdf.

[29] P. F. Whiting, A. W. Rutjes, M. E. Westwood et al., "QUADAS-2: a revised tool for the quality assessment of diagnostic accuracy studies," Annals of Internal Medicine, vol. 155, no. 8, pp. 529-536, 2011.

[30] X. Zhou, Y. Li, X. Jiang et al., "Intra-individual comparison of 18F-PSMA-1007 and 18F-FDG PET/CT in the evaluation of patients with prostate cancer," Frontiers in oncology, vol. 10, Article ID 585213, 2020.

[31] Y. Shi, L. Xu, Y. Zhu, Y. Wang, R. Chen, and J. Liu, "Use of 68Ga-PSMA-11 and 18F-FDG PET-CT dual-tracer to differentiate between lymph node metastases and ganglia," Frontiers in Oncology, vol. 11, Article ID 646110, 2021.

[32] R. Chen, Y. Wang, Y. Shi et al., "Diagnostic value of (18)FFDG PET/CT in patients with biochemical recurrent prostate cancer and negative (68)Ga-PSMA PET/CT," European Journal of Nuclear Medicine and Molecular Imaging, vol. 48, no. 9, pp. 2970-2977, 2021.

[33] B. Wang, C. Liu, Y. Wei et al., "A prospective trial of $68 \mathrm{Ga}$ PSMA and 18F-FDG PET/CT in nonmetastatic prostate cancer patients with an early PSA progression during castration," Clinical Cancer Research, vol. 26, no. 17, pp. 4551-4558, 2020.

[34] L. M. McEwan, D. Wong, and J. Yaxley, "Flourodeoxyglucose positron emission tomography scan may be helpful in the case of ductal variant prostate cancer when prostate specific membrane antigen ligand positron emission tomography scan is negative," Journal of Medical Imaging and Radiation Oncology, vol. 61, no. 4, pp. 503-505, 2017.

[35] A. Kichloo, R. Amir, M. Aljadah et al., "FDG-PET versus PSMA-PET: a patient with prostate cancer," Journal of investigative medicine high impact case reports, vol. 8, 2020.

[36] T. Cakir, S. Guven, B. Muezzinoglu, C. Erol, M. Y. Boz, and T. Atasever, "Intense fluoro-2-deoxyglucose uptake in highgrade prostatic adenocarcinoma with negligible prostatespecific membrane antigen expression," Urology Annals, vol. 11, no. 4, pp. 439-442, 2019.

[37] S. Chen, S. K. Cheung, K.-N. Wong, K. K. Wong, and C.-L. Ho, "68Ga-DOTATOC and 68Ga-PSMA PET/CT unmasked a case of prostate cancer with neuroendocrine differentiation," Clinical Nuclear Medicine, vol. 41, no. 12, pp. 959-960, 2016. 
[38] G. K. Parida, S. Tripathy, S. Datta Gupta et al., "Adenocarcinoma prostate with neuroendocrine differentiation," Clinical Nuclear Medicine, vol. 43, no. 4, pp. 248-249, 2018.

[39] D. Shetty, H. Loh, C. Bui, R. Mansberg, A. Hadjashrafi, and V. Do, "Non-prostate-specific membrane antigen-avid metastatic lung nodule from primary prostatic adenocarcinoma," Clinical Nuclear Medicine, vol. 41, no. 10, pp. 776-778, 2016.

[40] R. Parghane and S. Basu, "Small cell transformation of metastatic prostate adenocarcinoma diagnosed by dual-tracer PET/CT (68Ga-PSMA and 18F-FDG): potential clinical utility in therapeutic decision making and treatment monitoring," Journal of Nuclear Medicine Technology, vol. 47, no. 1, pp. 85-87, 2019.

[41] P. M. Perez, T. A. Hope, S. C. Behr, A. Van Zante, E. J. Small, and R. R. Flavell, "Intertumoral heterogeneity of 18F-FDG and 68Ga-PSMA uptake in prostate cancer pulmonary metastases," Clinical Nuclear Medicine, vol. 44, no. 1, pp. e28-e32, 2019.

[42] L. Geraldo, F. Ceci, C. Uprimny, D. Kendler, and I. Virgolini, "Detection of sarcomatoid lung metastasis with 68GA-PSMA PET/CT in a patient with prostate cancer," Clinical Nuclear Medicine, vol. 41, no. 5, pp. 421-422, 2016.

[43] G. Polverari, F. Ceci, M. Allen-Auerbach et al., "Solitary mucinous prostate adenocarcinoma lung metastasis detected by Ga-68-PSMA-11 PET/CT," Clinical Genitourinary Cancer, vol. 17, no. 1, pp. E53-E55, 2019.

[44] M. A. Queiroz, P. Viana, A. Santos, D. Bastos, E. Etchebehere, and G. Cerri, "Clinical impact of Ga-68-PSMA PET/CT in a patient with biochemical recurrence of prostate cancer," Clinical Nuclear Medicine, vol. 41, no. 9, pp. E417-E419, 2016.

[45] I. Sarikaya, A. Elgazzar, A. Sarikaya, and M. Alfeeli, "Fluorodeoxyglucose, sodium fluoride, and prostate-specific membrane antigen positron emission tomography studies for treatment response assessment in prostate cancer," World Journal of Nuclear Medicine, vol. 17, no. 3, pp. 207-210, 2018.

[46] S. McGeorge, R. Phillips, N. J. Rukin, J. Reynolds, and M. J. Roberts, "Solitary castrate-resistant prostate cancer metastasis to adrenal gland with concordant intense avidity on PSMA and FDG PET," Urology Case Reports, vol. 38, Article ID 101696, 2021.

[47] C. J. Sweeney, Y.-H. Chen, M. Carducci et al., "Chemohormonal therapy in metastatic hormone-sensitive prostate cancer," New England Journal of Medicine, vol. 373, no. 8, pp. 737-746, 2015.

[48] D. A. Palma, R. Olson, S. Harrow et al., "Stereotactic ablative radiotherapy versus standard of care palliative treatment in patients with oligometastatic cancers (SABR-COMET): a randomised, phase 2, open-label trial," The Lancet, vol. 393, no. 10185, pp. 2051-2058, 2019.

[49] R. Phillips, W. Y. Shi, M. Deek et al., "Outcomes of observation vs stereotactic ablative radiation for oligometastatic prostate cancer," JAMA Oncology, vol. 6, no. 5, pp. 650-659, 2020.

[50] S. Suman, R. V. Parghane, A. Joshi et al., "Therapeutic efficacy, prognostic variables and clinical outcome of 177Lu-PSMA-617 PRLT in progressive mCRPC following multiple lines of treatment: prognostic implications of high FDG uptake on dual tracer PET-CT vis-à-vis Gleason score in such cohort," British Journal of Radiology, vol. 92, no. 1104, 2019.

[51] S. P. Thang, J. Violet, S. Sandhu et al., "Poor outcomes for patients with metastatic castration-resistant prostate cancer with low prostate-specific membrane antigen (PSMA) expression deemed ineligible for 177Lu-labelled PSMA radioligand therapy," European Urology Oncology, vol. 2, no. 6, pp. 670-676, 2019.

[52] F. Rosar, K. Ribbat, M. Ries et al., "Neuron-specific enolase has potential value as a biomarker for $[18 \mathrm{~F}] \mathrm{FDG} /[68 \mathrm{Ga}] \mathrm{Ga}$ PSMA-11 PET mismatch findings in advanced mCRPC patients," EJNMMI Research, vol. 10, no. 1, 2020.

[53] H. Beltran, D. Prandi, J. M. Mosquera et al., "Divergent clonal evolution of castration-resistant neuroendocrine prostate cancer," Nature Medicine, vol. 22, no. 3, pp. 298-305, 2016.

[54] M. K. Bakht, J. M. Lovnicki, J. Tubman et al., "Differential expression of glucose transporters and hexokinases in prostate cancer with a neuroendocrine gene signature: a mechanistic perspective for 18F-FDG imaging of PSMA-suppressed tumors," Journal of Nuclear Medicine, vol. 61, no. 6, pp. 904-910, 2020.

[55] E. Acar and G. Ç. Kaya, "18F-FDG, 68Ga-DOTATATE and 68Ga-PSMA positive metastatic large cell neuroendocrine prostate tumor," Clinical Nuclear Medicine, vol. 44, no. 1, pp. 53-54, 2019.

[56] V. Prati, F. Munoz, O. Dal Canton et al., "68GA-PSMA: high sensibility, specificity and detection rate tracer even with low PSA level: case report," Anticancer Research, vol. 37, no. 4, pp. 2058-2059, 2017.

[57] F. H. Cornelis, J. C. Durack, M. J. Morris, H. I. Scher, and S. B. Solomon, "Effective prostate-specific membrane antigenbased 18F-DCFPyL-Guided cryoablation of a single positive site in a patient believed to be more metastatic on 11C-choline PET/CT," Clinical Nuclear Medicine, vol. 42, no. 12, pp. e516-e518, 2017.

[58] B. L. Liu, J. J. Hong, K. Tang, J. Lin, and X. W. Zheng, "18FPSMA-1007 uptake in pulmonary lymphangitic carcinomatosis metastasis from prostate cancer," Clinical Nuclear Medicine, vol. 46, no. 7, pp. e355-e357, 2021.

[59] C. Soydal, E. Ozkan, H. Yerlikaya, G. Utkan, and O. N. Kucuk, "Widespread metastatic prostate carcinoma shown by $68 \mathrm{Ga}$ PSMA PET/CT,” Clinical Nuclear Medicine, vol. 41, no. 6, pp. e294-e295, 2016. 